\title{
Reducing Host Interference from Spread Spectrum Watermarking
}

\author{
Saeed Sedghi • Yôiti Suzuki
}

Received: 31 May 2007 / Revised: 9 October 2007 / Published online: 28 February 2008

(C) The Author(s) 2008

\begin{abstract}
Spread spectrum watermarking (SSW) is one of the most promising watermarking techniques. While SSW is very robust against additive noise, it suffers from an inherent noise. The noise results from host interference on detection of the embedded hidden information. The host interference is one of the main reasons for the relatively low embedding rate in SSW and its reduction is important. In this paper, a new method for use in an SSW detector is proposed to reduce the host interference. The proposed method is based on the law of large numbers and symbol detection of embedded information, instead of bit detection. Since the method is based on the detection of symbols longer than one bit, the detector can employ PN sequences with a larger length, resulting in decrease of the interference. The symbol error rate using this algorithm is calculated employing the maximum a posteriori criterion. Comparison of bit error rates between the modified SSW algorithm and conventional bit detection shows that the modified algorithm is much more robust against the host interference.
\end{abstract}

Keywords Spread spectrum watermarking $\cdot$ Host interference $\cdot$ Symbol detection . Bit error rate

\section{Introduction}

During the last decade many algorithms for watermarking systems have been proposed to hide the existence of information into another signal as host. Spread spec-

\footnotetext{
S. Sedghi $(\bowtie)$

Department of Electrical Engineering, Ferdowsi University of Mashhad, Mashhad, Iran e-mail: saeed_sedghi@ieee.org
}

Y. Suzuki

Research Institute of Electrical Communication, Tohoku University, Sendai, Japan e-mail: yoh@ais.riec.tohoku.ac.jp 
trum watermarking (SSW) has been gaining a reputation as the most promising algorithm for both audio and image watermarking. The reasons for the popularity of SSW for watermarking techniques is that it is very robust against attacks including signal processing, noise and steganalysis [2] and it also has a high degree of perceptual transparency. Moreover, SSW is very flexible for the transmission of information in different situations since it flattens the spectrum of hidden information.

However, SSW suffers from some disadvantages. One of the most important ones is the host interference noise. That is, the host signal for SSW hinders correct detection of the embedded hidden information. Hence, even for situations where the watermark channel does not introduce any attack, correct detection of hidden information is limited. The host interference is an inherent noise in SSW algorithms and is the main reason for the relatively low embedding rate in SSW [7].

Reducing the host interference is, therefore, one of the major efforts in improving the SSW algorithm. Zhong et al. [9] proposed an idea for compensating the interference in the embedder of a DCT-based image SSW multiplicative scheme. First, they calculated the optimum decision threshold to reduce the interference and designed the new embedder according to the optimum decision rules for the detector. Gkizeli et al. [3] suggested use of the minimum-eigenvalue eigenvector of the host data in the transform domain for reducing the interference. J. Hernandez [5] and Cheng et al. [1] designed an optimum detector for additive SSW in the DCT-domain-based SSW by utilizing the probability density function of the host signal.

In this paper, a novel approach based on modifying the SSW detection strategy is proposed to reduce the host interference. The approach is applicable for the image and audio SSW, but we focus our calculations and results on one-dimensional signals, i.e. typically audio signals. The SSW scheme in this paper is considered additive and the calculations are much less intensive than those in the aforementioned methods. Moreover, there is not any change to the embedding strategy; the modifications are only for the detection strategy.

The rest of the paper is organized as follows. In Sect. 2, the SSW scheme and the host interference noise are introduced. Section 3 describes the new method and discusses its efficiency by evaluating symbol error rates. Sections 4 and 5 respectively present the bit detection algorithm and computer simulation results. The conclusion is given in Sect. 6.

\section{Spread Spectrum Watermarking}

In this section the SSW embedding and detection strategy is briefly reviewed for specifying the considered SSW algorithm, introducing the host interference and also the notation. Since we focus on host interference, the SSW system in the case where the channel does not introduce any attack is considered. However, it is possible to extend the results to the condition where the channel introduces attack.

The structure of the SSW approach is illustrated in Fig. 1. The frequency spectrum of hidden information $M$, is spread using a pseudo-random noise (PN) sequence with the length of $N$ chips $P=[p(1), \ldots, p(N)]$, to produce watermark signal $W$ as follows:

$$
W_{i}=M_{i} P, \quad i=1,2, \ldots,
$$




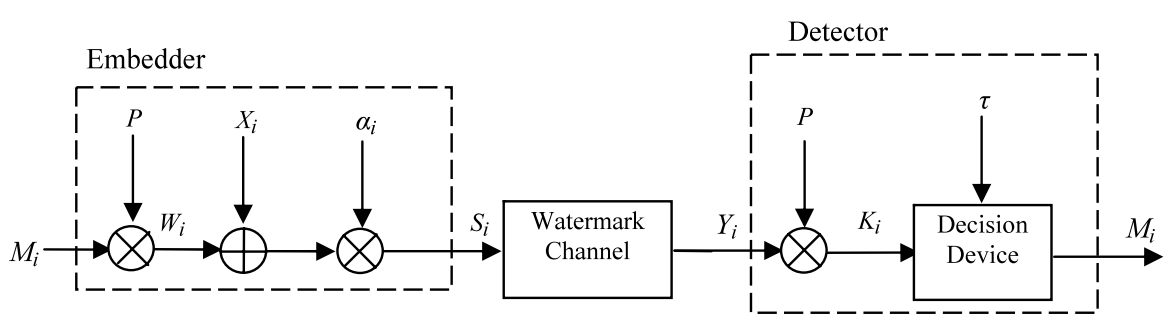

Fig. 1 SSW structure of the embedding and the detector

where $W_{i}=\left[w_{i}(1), \ldots, w_{i}(N)\right]$ is the $i$-th vector of $W$ and $M_{i} \in\{-1,1\}$ is the $i$-th bit of $M$. Then, the watermarked signal $S$ is produced as follows:

$$
S_{i}=\alpha_{i} W_{i}+X_{i}
$$

where $X_{i}=\left[x_{i}(1), \ldots, x_{i}(N)\right]$ is the $i$-th vector of the host signal $X, S_{i}=$ $\left[s_{i}(1), \ldots, s_{i}(N)\right]$ is the $i$-th vector of $S$ and $\alpha_{i} \in[0,1]$ is the parameter to control the intensity of the watermark.

At the receiving side, the detector will detect embedded $M_{i}$ in accordance with the correlation property of the $\mathrm{PN}$-sequence. Considering the property, cross correlation between the signal received by the detector $Y$ and the pseudo-random signal $P$ is denoted as follows:

$$
K_{i}=\frac{1}{N} Y_{i} P^{T}=\frac{1}{N}\left(\alpha_{i} W_{i}+X_{i}\right) P^{T}=\alpha_{i} M_{i}+K_{i x},
$$

where $Y_{i}=\left[y_{i}(1), \ldots, y_{i}(N)\right]$ is the $i$-th vector of $Y$ and $K_{i x}$ is as follows:

$$
K_{i x}=\frac{1}{N} \sum_{k=1}^{N} x_{i}(k) p(k) .
$$

By comparing $K_{i}$ at a decision device with a threshold $\tau, M_{i}$ will be detected. Since $M_{i} \in\{-1,1\}$ and $K_{i x}$ is a zero-mean signal (since $P$ is a zero-mean signal), $\tau$ is considered to be zero in traditional SSW algorithms.

As (4) shows, the signal $K_{i x}$ interferes with correct detection of $M_{i}$. Moreover, since we considered SSW in the case where the watermark channel does not introduce any attack, (4) shows that even in this case $K_{i x}$ will remain and interfere with the detection of $M_{i}$. Since $K_{i x}$ depends only on $X$ it is called the host interference and is an inherent noise in SSW.

\section{Reducing the Host Interference}

Equation (4) shows that the host interference, $K_{i x}$, depends on $N$ and $X$ only. Obviously, although employing $X$ with a smaller power would reduce $K_{i x}$, it may introduce some perceptual distortions. Thus, we should focus on another effective parameter, $N$. In accordance with the law of large numbers [4], as $N$ grows larger, $K_{i x}$ 
converges to its expectation as follows:

$$
K_{i x}=\frac{1}{N} \sum_{k=1}^{N} x_{i}(k) p(k) \stackrel{\text { Larger } N}{\longrightarrow} E\left(K_{i x}\right)=0,
$$

where $E($.$) stands for the expectation function and its convergence to zero is derived$ from the fact that $E(X)=E(P)=0$. Hence, using $P$ with larger $N$ is a suitable way to reduce $K_{i x}$. However, using $P$ with larger $N$ will decrease the embedding rate and there will be a trade-off between the host interference and the embedding rate.

In this section, reducing $K_{i x}$ without a decrease in the embedding rate is discussed. This aim could be achieved if only the detector increases the length of $P$ for the detection. An increase in the length of $P$ is possible if the detector performs the detection symbol by symbol instead of bit by bit. In other words, instead of using correlation between $Y_{i}$ and $P$ to detect $M_{i}$, the detector uses correlation between $P_{\mathrm{s}}$, for which elements $p_{\mathrm{s}}(l)$ are specified as follows, and $Y_{i}, \ldots, Y_{i-(m-1)}$ to detect $M_{i}, \ldots, M_{i-(m-1)}$ simultaneously.

$$
p_{\mathrm{s}}(l)=p_{\mathrm{s}}(l+N)=\cdots=p_{\mathrm{s}}[l+(m-1) N]=p(l), \quad l=1, \ldots, N,
$$

where $m$ is an integer defining the increase in the length of $P$ and $m \geq 2$. Hence, using the detection strategy of symbol detection the cross correlation for the symbol detection would be as follows:

$$
K_{i}=\frac{1}{m N}\left[W_{i-(m-1)}+X_{i-(m-1)}+\cdots+W_{i}+X_{i}\right] P_{\mathrm{s}} .
$$

And the host interference is as follows:

$$
K_{i x}=\frac{1}{m N} \sum_{k=1}^{N}\left[x_{i}(k)+x_{i-1}(k)+\cdots+x_{i-(m-1)}(k)\right] p(k) .
$$

Therefore, the denominator to calculate $K_{i x}$ is increased from $N$ to $m N$ and according to the law of large numbers the host interference would be decreased.

In order to examine the efficiency of the proposed method, a symbol error rate (SER) evaluation of the detected hidden information symbols should be performed. In this paper an SER for the detection of symbols equal to two bits, i.e. $m=2$, is calculated to examine the effectiveness of the proposed method. Therefore, in this case the correlation at the detector is performed between the received $Y_{i}$ and $Y_{i-1}$ and $P_{\mathrm{S}}$ as follows:

$$
\begin{aligned}
K_{i} & =\frac{1}{2 N}\left[W_{i-1}+X_{i-1}+W_{i}+X_{i}\right] P_{\mathrm{s}}^{T} \\
& =\frac{1}{2}\left(M_{i-1}+M_{i}\right)+\frac{1}{2 N} \sum_{k=1}^{N}\left[x_{i}(k)+x_{i-1}(k)\right] p(k) .
\end{aligned}
$$

In the following notation, the detected symbol $(1 / 2)\left(M_{i}+M_{i-1}\right)$ is called $L_{i}$ and $\left|L_{i}\right|$ denotes the value of $L_{i} . L_{i}^{1}, L_{i}^{2}$ and $L_{i}^{3}$ denote $\left|L_{i}\right|$ equal to $-1,0$, and 1 
respectively. $K_{i}^{1}, K_{i}^{2}$ and $K_{i}^{3}$ are the values of $K_{i}$ in the case where $L_{i}^{1}, L_{i}^{2}$ and $L_{i}^{3}$ have been transmitted respectively and $P_{\mathrm{r}}$ is the symbol probability.

The following evaluations of SER are based on the maximum a posteriori (MAP) criteria for the detection of $L_{i}$ [8]. Since $M_{i} \in\{-1,1\}$, the following are obtained:

$$
P_{\mathrm{r}}\left(\left|L_{i}\right|=1\right)=\frac{1}{4}, \quad P_{\mathrm{r}}\left(\left|L_{i}\right|=0\right)=\frac{1}{2}, \quad P_{\mathrm{r}}\left(\left|L_{i}\right|=-1\right)=\frac{1}{4} .
$$

Also, the value of $K_{i}$ is as follows:

$$
K_{i}=\left|L_{i}\right|+K_{i x}
$$

Using MAP criteria to minimize SER, the symbol will be detected so that it maximizes the mutual probability of $K_{i}$ and $L_{i}$ at the detector as follows:

$$
P_{\mathrm{r}}\left(K_{i}, L_{i}^{j}\right)=P_{\mathrm{r}}\left(K_{i} \mid L_{i}^{j}\right) P_{\mathrm{r}}\left(L_{i}=L_{i}^{j}\right), \quad j=1,2,3,
$$

where $P_{\mathrm{r}}\left(K_{i} \mid L_{i}^{j}\right)$ denotes the probability of $K_{i}$ when $\left|L_{i}\right|=L_{i}^{j}, j=1,2,3$. In this case, the conditional probabilities are as follows:

$$
P_{\mathrm{r}}\left(K_{i} \mid L_{i}^{j}\right)=\frac{1}{\sqrt{2 \pi} \sigma_{K_{i x}}} \exp \left[-\frac{\left(K_{i}-L_{i}^{j}\right)^{2}}{2 \sigma_{K_{i x}}^{2}}\right], \quad j=1,2,3 .
$$

Decision of the transmitted symbol value from the received symbol should be performed by comparison with a threshold decision. Calculation of the threshold between the symbols $L_{i}^{2}$ and $L_{i}^{1}$ could be performed in the following way. If

$$
\frac{P_{\mathrm{r}}\left(K_{i}, L_{i}^{1}\right)}{P_{\mathrm{r}}\left(K_{i}, L_{i}^{2}\right)}>1
$$

it should be concluded that $L_{i}^{1}$ has been transmitted. Since an audio signal could be considered as a Gaussian distribution, $K_{i x}$ has a Gaussian distribution. Therefore, (14) is extended as follows:

$$
\frac{1}{2} \exp \left[-\frac{\left(K_{i}-1\right)^{2}}{2 \sigma_{K_{i x}}^{2}}+\frac{\left(K_{i}\right)^{2}}{2 \sigma_{K_{i x}}^{2}}\right]>1 \Rightarrow K_{i} \geq \frac{1}{2} \sigma_{K_{i x}}^{2} \operatorname{Ln} 2+1 .
$$

Note that it is assumed that the error probability of detection between the symbols $L_{i}^{3}$ and $L_{i}^{1}$ is very small in comparison with the error probability between $L_{i}^{1}$ and $L_{i}^{2}$ and therefore it can be ignored. Extending (14), the threshold would be calculated as follows:

$$
\sigma_{K_{i x}}^{2}=\frac{\operatorname{Pow}(X)}{\operatorname{Pow}(W) .2 N} \Rightarrow K_{i} \geq \frac{\operatorname{Ln}(2)}{4 N} H W R+1=C,
$$

where Pow(.) presents power of the signal and $H W R$ is the host-to-watermark ratio as follows:

$$
H W R=\frac{\operatorname{Pow}(X)}{\operatorname{Pow}(W)}
$$


Fig. 2 Signal space diagram of symbol detection

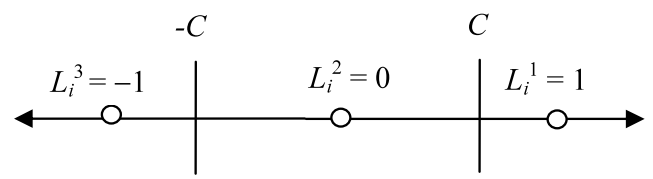

It is obvious that the threshold between the symbols $L_{i}^{2}$ and $L_{i}^{3}$ is $-C$, because $P_{\mathrm{r}}\left(L_{i}^{1}\right)=P_{\mathrm{r}}\left(L_{i}^{3}\right)$ and their distance to $L_{i}^{2}$ is the same. The signal space diagram of the detected symbols and the decision thresholds is illustrated in Fig. 2.

Employing the strategy of symbol detection, the SER is calculated as follows:

$$
P_{\mathrm{e}}=\sum_{m=1, m \neq n}^{3} P_{\mathrm{r}}\left(L_{i}^{m} \mid L_{i}^{n}\right) P_{\mathrm{r}}\left(L_{i}^{n}\right),
$$

where $P_{\mathrm{e}}$ is the symbol error rate.

Since the SER for the detection of $L_{i}^{3}$ when the symbol $L_{i}^{1}$ is transmitted and vice versa is very small, it can be ignored. Hence, the SER is calculated as follows:

$$
\begin{aligned}
P_{\mathrm{e}}= & \left.P_{\mathrm{r}}\left(\left|K_{i x}\right|>C \mid L_{i}^{2}\right) P_{\mathrm{r}}\left(L_{i}^{2}\right)+P_{\mathrm{r}}\left(K_{i x}<-(1-C) \mid L_{i}^{1}\right) P_{\mathrm{r}}\left(L_{i}^{1}\right)\right) \\
& +P_{\mathrm{r}}\left(K_{i x}>(1-C) \mid L_{i}^{3}\right) P_{\mathrm{r}}\left(L_{i}^{3}\right) .
\end{aligned}
$$

Finally, the formula for calculating the SER can be obtained as follows:

$$
P_{\mathrm{e}}=\left\{\frac{1}{2} Q\left[\sqrt{\frac{N}{H W R}+\operatorname{Ln}(2)}\right]+\frac{1}{4} Q\left[\sqrt{\frac{N}{H W R}-\operatorname{Ln}(2)}\right]\right\},
$$

where $Q($.$) is the complementary error function.$

\section{Bit Detection of Hidden Information}

We proposed an approach based on symbol detection of hidden information for reducing the host interference. However, there will be a question as to how the detector can detect bits of the detected symbol. In this section, bit detection from the detected symbols of 2-bits long is examined.

Obviously, if the detected $L_{i}$ is 1 or -1 , both $M_{i}$ and $M_{i-1}$ are 1 or -1 respectively. Moreover, if the detected $L_{i}$ is 0 , it specifies that $M_{i}$ and $M_{i-1}$ are of opposite signs. Thus, each of the two bits should be detected separately using correlation between $Y_{i}$ and $P$ and correlation between $Y_{i-1}$ and $P$. Since the detector knows that $M_{i}$ and $M_{i-1}$ are of opposite signs, the false detection occurs when the both bits are wrongly detected. It should be mentioned that this strategy is not efficient and the detector should perform more computations for the detection.

The other way that the bit detection could be performed is by using the following modification at the embedder: if the embedder embeds $M_{0}$ as a known bit to the detector, the detected $L_{1}$ contains $M_{0}$, which is known to the detector, and $M_{1}$, which is the first bit of the hidden information. Therefore, by the detection of $L_{1}, M_{1}$ will 
Table 1 BER and SER versus different $N$ values

\begin{tabular}{lllllllll}
\hline$N$ & 31 & 63 & 127 & 255 & 511 & 1023 & 2047 & 4095 \\
\hline BER & 0.30938 & 0.1473 & 0.03963 & 0.0035 & $3.67 \times 10^{-5}$ & $5.23 \times 10^{-9}$ & $1.45 \times 10^{-16}$ & $1.55 \times 10^{-31}$ \\
SER & 0.21726 & 0.0807 & 0.02032 & 0.0017 & $1.84 \times 10^{-5}$ & $2.61 \times 10^{-9}$ & $7.26 \times 10^{-17}$ & $7.75 \times 10^{-32}$ \\
\hline
\end{tabular}

be detected automatically. Also, the detected symbol $L_{2}$ contains $M_{1}$ and $M_{2}$. Since $M_{1}$ was previously detected, $M_{2}$ will be detected automatically. Therefore, if this procedure is repeated, the symbol detection will be performed using $M_{i-1}$, which has been detected in the previous detection, and $M_{i}$.

Although using the latter method requires fewer computations in comparison with the former, it has the disadvantage of error propagation. If one bit is wrongly detected, the wrong detection will be propagated to the other bits. Hence, it is better to use the latter only when the SER is very low. Furthermore, the embedder should repeat embedding of the known bit to the detector $M_{0}$ after several bits of $M$ in order to limit the propagation of the error.

\section{Simulation Results}

In this section, employing computer simulations, the performance of our proposed detection algorithm is examined. A comparison of the host interference between the bit detection strategy and the symbol detection strategy is illustrated in Fig. 3. The result shown in this figure is obtained for a music SSW using the parameters $N=1023$ and $H W R=18 \mathrm{~dB}$. As shown in the figure, the symbol detection strategy reduces the host interference remarkably.

The formula expressing the bit error rate (BER) for bit-by-bit detection as in traditional SSW is as follows [6]:

$$
P_{\mathrm{b}}=Q\left(\sqrt{\frac{N}{H W R}}\right),
$$

where $P_{\mathrm{b}}$ denotes BER. As (20) and (21) show, SER and BER depend on $N$ and $H W R$. Hence, the effect of both parameters should be examined. The comparison of BER and SER for various $N$ 's when $H W R=16 \mathrm{~dB}$ is illustrated in Table 1 . This table shows that SER is lower than BER for all the $N$ 's and as $N$ grows large, the ratio between SER and BER becomes larger.

Examining SER versus different values of $H W R$ is illustrated in Fig. 4. The simulation is performed to show the effect of $H W R$ on SER. The range of $H W R$ used in this figure is between $30(14.7 \mathrm{~dB})$ and $90(19.5 \mathrm{~dB})$. As the figure depicts, SER is below BER for all HWRs and the distance between SER and BER is larger as $H W R$ increases.

The method from the frequency domain point of view is investigated in Fig. 5. This figure illustrates the power spectral density (PSD) of a frame of speech signal which is used as a host signal, the PSD of the host after correlation with $P$, and also the PSD after the correlation with $P_{\mathrm{s}}$. As Fig. 5 shows, the PSD of the host signal, which is 


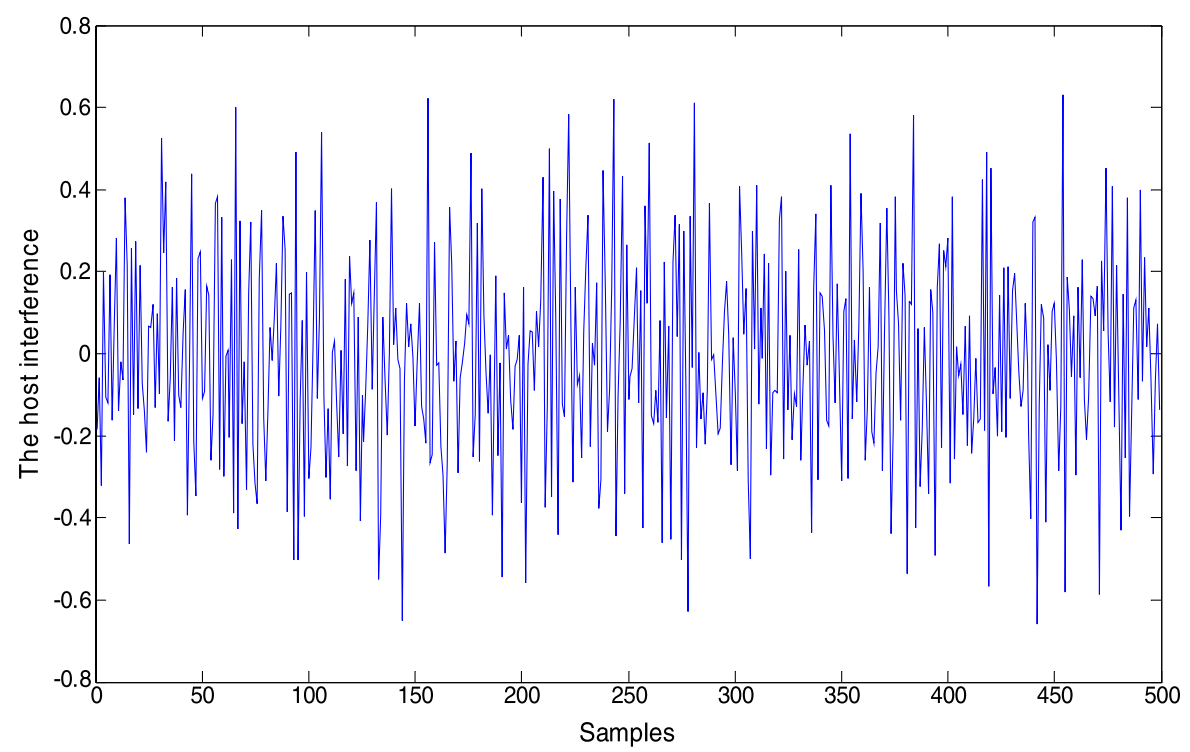

(a)

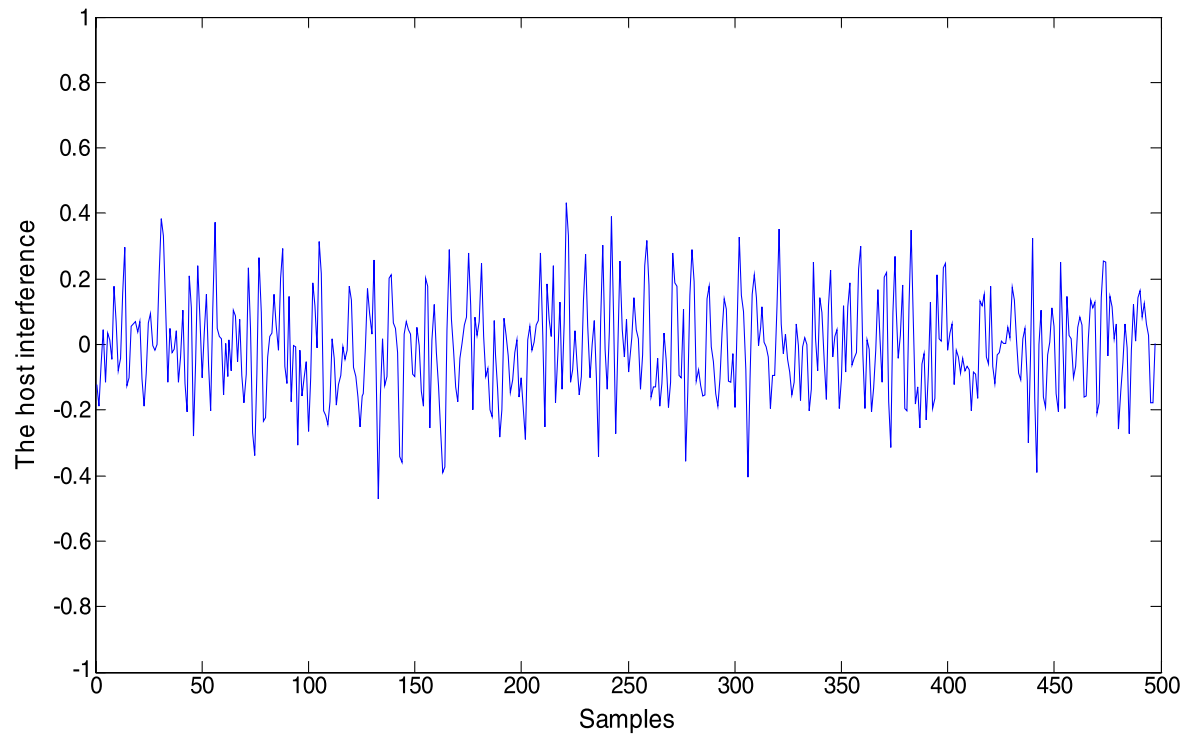

(b)

Fig. 3 The host interference for a music SSW for (a) bit detection strategy, (b) symbol detection strategy

strongly colored originally, is well whitened after taking the cross correlation of the host by $P$. Also it shows that after the cross correlation by $P_{\mathrm{s}}$ the PSD of the host is even whiter than when the correlation was performed using $P$. This figure shows how the proposed method behaves in the frequency domain. Since the symbol detection 


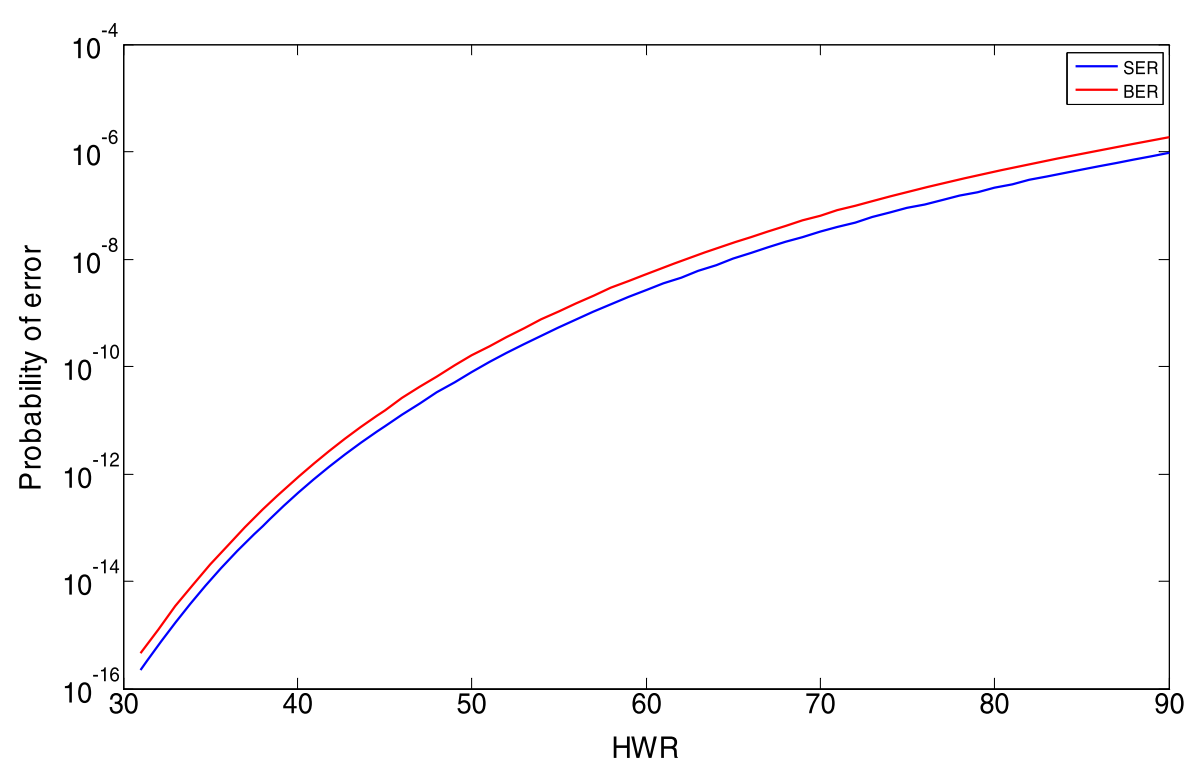

Fig. 4 BER and SER with different $H W R$

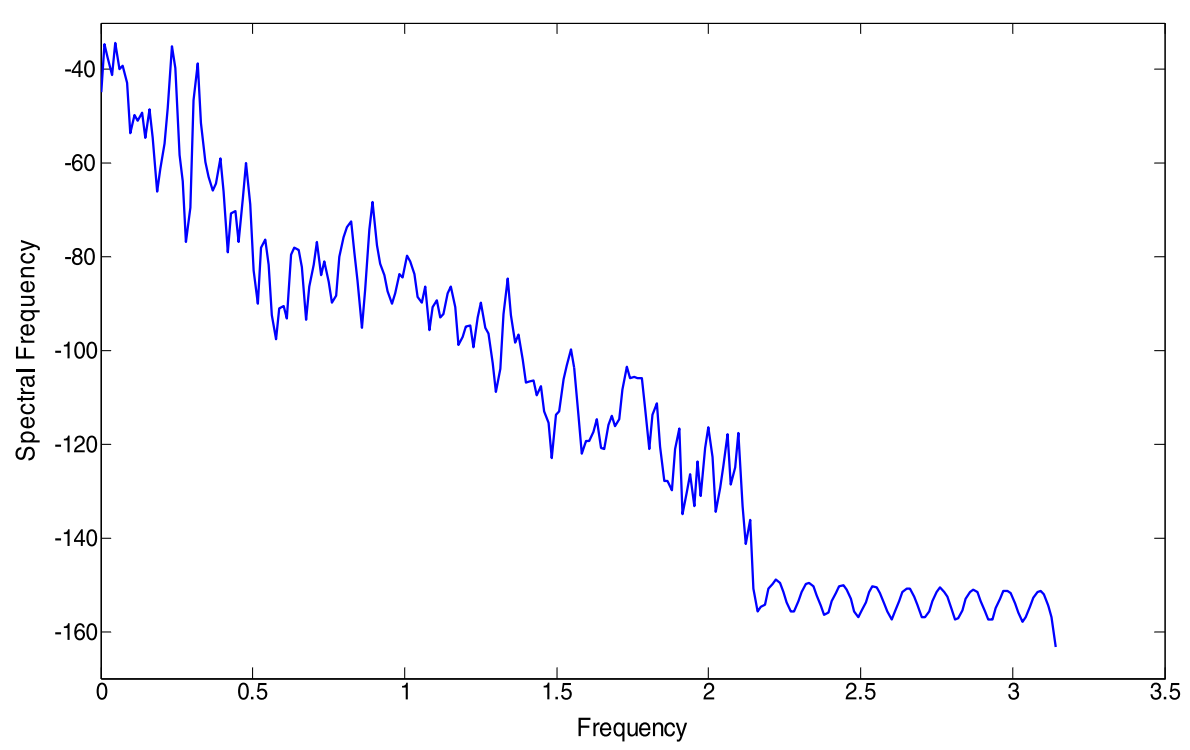

(a)

Fig. 5 The effect of symbol detection in frequency domain: (a) the PSD of host, (b) the PSD of host after correlation with $P,(\mathbf{c})$ the PSD of host after correlation with $P_{\mathrm{S}}$

strategy realizes better whitening than the bit detection strategy, symbol detection has lower SER than bit detection. 


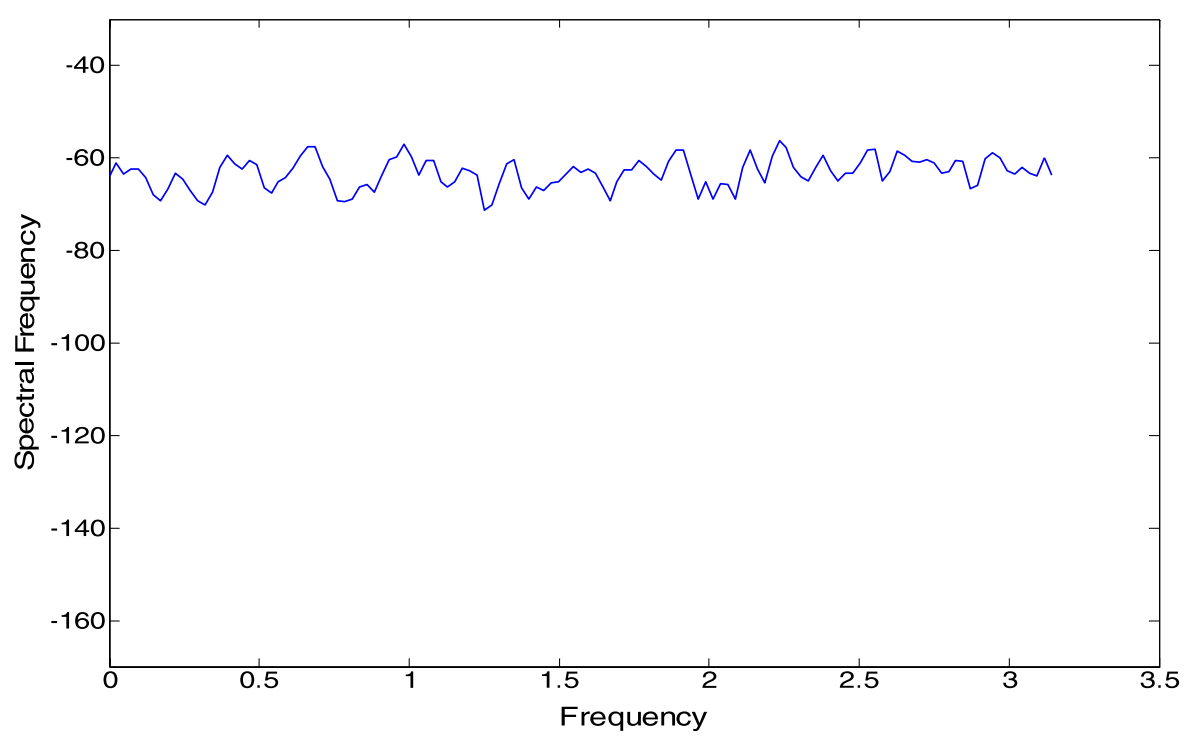

(b)

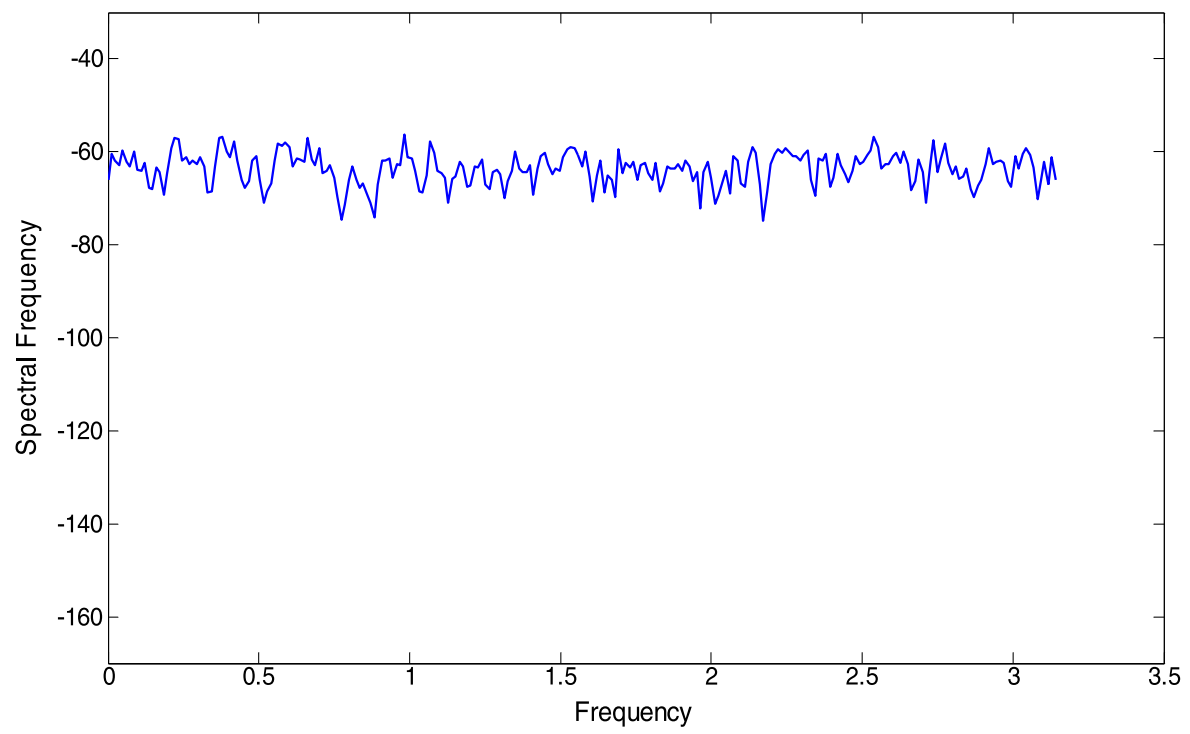

(c)

Fig. 5 (Continued)

\section{Conclusion}

A new method to reduce the host interference in an SSW detector was presented. The idea is based on the symbol detection of hidden information instead of the bit detection. Since the method is based on the detection of symbols longer than one bit, the detector can employ PN sequences with a larger length, resulting in a decrease in the 
interference. Comparison of BERs between the proposed SSW algorithm and conventional bit detection showed that the host interference can be remarkably reduced with the proposed method. Investigation of the proposed method in the frequency domain shows that whitening of the host signal at the detector using the symbol detection strategy is better than that with the bit detection strategy. As a result, the symbol detection strategy has lower detection error than the bit detection strategy.

Open Access This article is distributed under the terms of the Creative Commons Attribution Noncommercial License which permits any noncommercial use, distribution, and reproduction in any medium, provided the original author(s) and source are credited.

\section{References}

1. Cheng, Q., Huang, T.S.: An additive approach to transform-domain information hiding and optimum detection structure, IEEE Trans. Multimedia vol. 3, no. 9, 273-284, 2001.

2. Cox, I.J., Killian, J., Leighton, F.T., Shanmoon, T.: Secure spread spectrum watermarking for multimedia, IEEE Trans. Image Process. vol. 6, no. 12, 1673-1687, 1997.

3. Gkizeli, M., Pados, D.A., Medley, M.J.: Optimal signature design for spread spectrum steganography, IEEE Trans. Image Process. vol. 16, no. 2, 391-406, 2007.

4. Grimmett, G.R., Stirzaker, D.R.: Probability and Random Processes, 2nd Edition, Clarendon, Oxford, 1992. ISBN 0-19-853665-8.

5. Hernández, J.R., Amado, M., Pérez-González, F.: DCT-domain watermarking techniques for still images: Detector performance analysis and a new structure, IEEE Trans. Image Process. vol. 9, no. 1, 55-68, 2000.

6. Jitsumatsu, Y., Hattori, Y., Khan, T.A.: T, Kohda, Bit error rates of information and watermark bits in digital watermarking systems based on SS techniques, IEEE Proc. Spread Spectrum Techniques, 2004.

7. Moulin, P., Sullivan, J.O.: Information theoretic analysis of information hiding, IEEE Trans on Information Theory, 2003, pp. 563-593.

8. Proakis, J.G.: Digital Communications, 4th Edition, McGraw-Hill, New York, 2000.

9. Zhong, J., Huang, S.: An enhanced multiplicative spread spectrum watermarking scheme, IEEE Trans. Circuits Syst. Video Technol. vol. 16, no. 2, 1491-1506, 2006. 